\title{
Cost-Effectiveness of Multiple Sclerosis Disease-Modifying Therapies: A Systematic Review of the Literature
}

\author{
David Yamamoto ${ }^{1}$ and Jonathan D. Campbell ${ }^{2}$ \\ ${ }^{1}$ Department of Health Systems, Management, and Policy, Colorado School of Public Health, 13001 E 17th Place, \\ Aurora, CO 80045, USA \\ ${ }^{2}$ Department of Clinical Pharmacy, Skaggs School of Pharmacy and Pharmaceutical Sciences, University of Colorado, \\ Anschutz Medical Campus, Aurora, CO 80045, USA \\ Correspondence should be addressed to Jonathan D. Campbell, jon.campbell@ucdenver.edu
}

Received 10 August 2012; Accepted 31 October 2012

Academic Editor: Giovanni Savettieri

Copyright ( 2012 D. Yamamoto and J. D. Campbell. This is an open access article distributed under the Creative Commons Attribution License, which permits unrestricted use, distribution, and reproduction in any medium, provided the original work is properly cited.

Objective. To provide a current and comprehensive understanding of the cost-effectiveness of DMTs for the treatment of MS by quantitatively evaluating the quality of recent cost-effectiveness studies and exploring how the field has progressed from past recommendations. Methods. We assessed the quality of studies that met our systematic literature search criteria using the Quality of Health Economic Studies validated instrument. Results. Of the 82 studies that met our initial search criteria, we included 22 in this review. Four studies (18\%) achieved quality category 2, three studies (14\%) achieved quality category 3 , and 15 studies $(68 \%)$ achieved the highest quality category $4.91 \%$ of studies were simulation models. 13 studies (59\%) had quality-adjusted life years (QALYs) as the primary outcome measure, included a societal perspective in the analysis, and utilized time horizons of 10 years to lifetime. Conclusions. To continue to improve the cost-effectiveness evidence of DMTs, we recommend: lifetime horizons, societal perspectives, and QALYs; supplemental evidence with shorter horizons, payer perspectives, and clinical outcomes to inform multiple decision makers; development of modeling and input standards for comparability; head-to-head RCTs between DMTs and long-term prospective studies; and comprehensive cost-effectiveness studies that compare all appropriate DMTs.

\section{Introduction}

Multiple sclerosis (MS) is a chronic and debilitating inflammatory autoimmune disorder of the central nervous system that afflicts approximately 400,000 people in the United States and 2.1 million people worldwide $[1,2]$. MS is one of the most common contributors to neurological disability in young and middle-aged adults [3]. The peak age of onset is approximately 30 years, and the disease occurs in twice as many women as men [4]. About $85 \%$ of MS patients have the relapsing-remitting form of the disease [1]. Symptoms of MS include fatigue, impaired mobility, spasticity, pain, depression, cognitive impairment, sexual dysfunction, bowel and bladder dysfunction, vision and hearing problems, seizures, and difficulty swallowing [2]. The multifaceted symptoms of MS all are associated with economic burden, as well as an adverse impact on patients' quality of life.
Treatment of MS has advanced significantly over the past several decades. Historically, MS was treated in a solely supportive manner through symptomatic pharmacological treatment in the event of disease exacerbations (MS attacks), generally via powerful doses of short-course steroids [5]. However, starting in the 1990s the FDA approved the first disease-modifying therapies (DMTs) for the long-term treatment of the disease. These new biologics were intended to proactively manage and retard disease progression. DMTs have demonstrated favorable risk-benefit profiles for US and other jurisdictions' regulatory approval. Some DMTs have more significant benefits and risks than others. DMTs have provided a promising new means of managing this chronic and debilitating disease; however, they have also introduced greatly elevated, and rapidly increasing, costs to the treatment of MS [6]. Cost-of-illness studies in the early 2000s estimated that medication including DMTs accounted 
TABLE 1: Characteristics of disease-modifying therapies for multiple sclerosis.

\begin{tabular}{|c|c|c|c|c|c|}
\hline DMT brand name (Generic name) & Manufacturer & $\begin{array}{c}\text { FDA } \\
\text { approval year }\end{array}$ & $\begin{array}{c}\text { Dose frequency } \\
\text { administration [5] }\end{array}$ & $\begin{array}{c}2010 \text { Annual } \\
\text { Tx cost } \\
{[13,14]}\end{array}$ & $\begin{array}{l}\text { Significant risks listed } \\
\text { in package insert }\end{array}$ \\
\hline Betaseron [15] (IFN beta-1b) & $\begin{array}{c}\text { Bayer Health Care } \\
\text { Pharmaceuticals, Inc }\end{array}$ & 1993 & 250 ug 2 days SC & $\$ 38,369$ & \\
\hline Avonex [16] (IFN beta-1a) & Biogen Idec, Inc & 1996 & 30 ug weekly IM & $\$ 38,532$ & \\
\hline $\begin{array}{l}\text { Copaxone [17] (Glatiramer } \\
\text { acetate) }\end{array}$ & Teva Neuroscience, Inc & 1996 & $20 \mathrm{mg}$ daily SC & $\$ 42,940$ & \\
\hline Novantrone [18] (Mitoxantrone) & $\begin{array}{c}\text { Novartis } \\
\text { Pharmaceuticals } \\
\text { Corporation }\end{array}$ & 2000 & $\begin{array}{l}12 \mathrm{mg} / \mathrm{m}^{2} \\
3 \text { months IV }\end{array}$ & $\$ 6,344$ & Cardiotoxicity \\
\hline Rebif [19] (IFN beta-1a) & EMD Serono, Inc & 2000 & 44 ug $3 x$ weekly SC & $\$ 38,646$ & \\
\hline Tysabri [20] (Natalizumab) & $\begin{array}{l}\text { Elan Pharmaceuticals, } \\
\text { Inc and Biogen Idec, Inc }\end{array}$ & 2004 & $300 \mathrm{mg} 4$ weeks IV & $\$ 40,426$ & $\begin{array}{l}\text { Increased risk of } \\
\text { PML, } \\
\text { Elevated risk for } \\
\text { infections }\end{array}$ \\
\hline Extavia [21] (IFN beta-1b) & $\begin{array}{c}\text { Novartis } \\
\text { Pharmaceuticals } \\
\text { Corporation }\end{array}$ & 2009 & 250 ug 2 days SC & $\$ 38,368$ & \\
\hline Gilenya [22] (Fingolimod) & $\begin{array}{l}\text { Novartis Pharmaceutical } \\
\text { Corporation }\end{array}$ & 2010 & $0.5 \mathrm{mg}$ daily oral & $\$ 47,944$ & $\begin{array}{l}\text { Cardiotoxicity, } \\
\text { Elevated risk for } \\
\text { infections }\end{array}$ \\
\hline
\end{tabular}

IFN: Interferon; IM: intramuscular; IV: intravenous; PML: progressive multifocal leukoencephalopathy; SC: subcutaneous; Tx: treatment.

for $\sim 7 \%$ of overall costs as reported across similar studies in Germany [7], UK [8], Sweden [9], and Canada [10]. In 2004, Kobelt found that in the US DMTs had begun to account for $34 \%$ of total costs per MS patient each year, and over half of all direct medical costs [11]. Similarly, an Italian 2004 study suggested that $77 \%$ of direct medical costs were attributed to DMTs [12]. On average DMTs cost $\$ 16,050$ per MS-treated patient per year in 2004 dollars. By 2010, the current annual treatment costs had risen by two- to threefold over this 2004 average for all DMTs except mitoxantrone. The eight FDA approved DMTs as of August 2012 are characterized in Table 1.

Three review studies from 2003-4 focused on costeffectiveness of DMTs in the treatment of MS and included multiple current therapies [3, 23, 24]. In 2004, Flachenecker published a review that comprehensively studied all DMTs available at that time [25]. In 2006, Kobelt published a review and informational article on studies of MS therapies; however, the focus was mainly methodological and did not compile and compare costs and effectiveness results in concert to inform decision makers [26]. In 2006, Reickmann published a brief review on the socioeconomic aspects of neuroimmunological diseases focusing on MS [27]. Prior reviews came to very similar conclusions suggesting use of the societal perspective, appropriate long-term modeling to reflect the chronic nature of MS, and standard endpoints and modeling to determine the cost-effectiveness of DMTs. The authors also indicated a dearth of comprehensive and uniform prospective studies, which is important in further understanding the costs and outcomes associated with different DMTs.

Over the past ten years, there has been a lack of comprehensive and systematic reviews of multiple sclerosis cost-effectiveness studies. Previous reviews did not address DMTs approved in recent years (natalizumab or fingolimod) and none have been recent and comprehensive enough to detail the current state of the cost-effectiveness of DMTs in treating multiple sclerosis. The aim of this systematic review was to provide a current and comprehensive understanding of the cost-effectiveness of DMTs for the treatment of multiple sclerosis by quantitatively evaluating the quality of cost-effectiveness studies and exploring how the multiple sclerosis cost-effectiveness field has progressed from past recommendations.

\section{Methods}

2.1. Cost-Effectiveness Analyses. Cost-effectiveness analysis (CEA) is a full form of health economic analysis, where both costs and consequences (outcomes) of health programs or treatments are examined. CEA is used in situations where decision makers are considering a limited range of options within a given field, and within a given operating budget [28]. CEA is used to compare the benefits and costs of a program, intervention, or treatment to its next best alternative in order to determine whether it is of sufficient value to adopt or reimburse [29]. The primary output of a costeffectiveness study is the incremental cost-effectiveness ratio (ICER), which compares two alternative interventions' average costs $\left(C_{1}\right.$ and $\left.C_{2}\right)$ and effects $\left(E_{1}\right.$ and $\left.E_{2}\right)$ in the form of the following ratio:

$$
\frac{\left(C_{1}-C_{2}\right)}{\left(E_{1}-E_{2}\right)} .
$$

The effectiveness measure chosen should properly reflect a final output, rather than a secondary or intermediate output. 


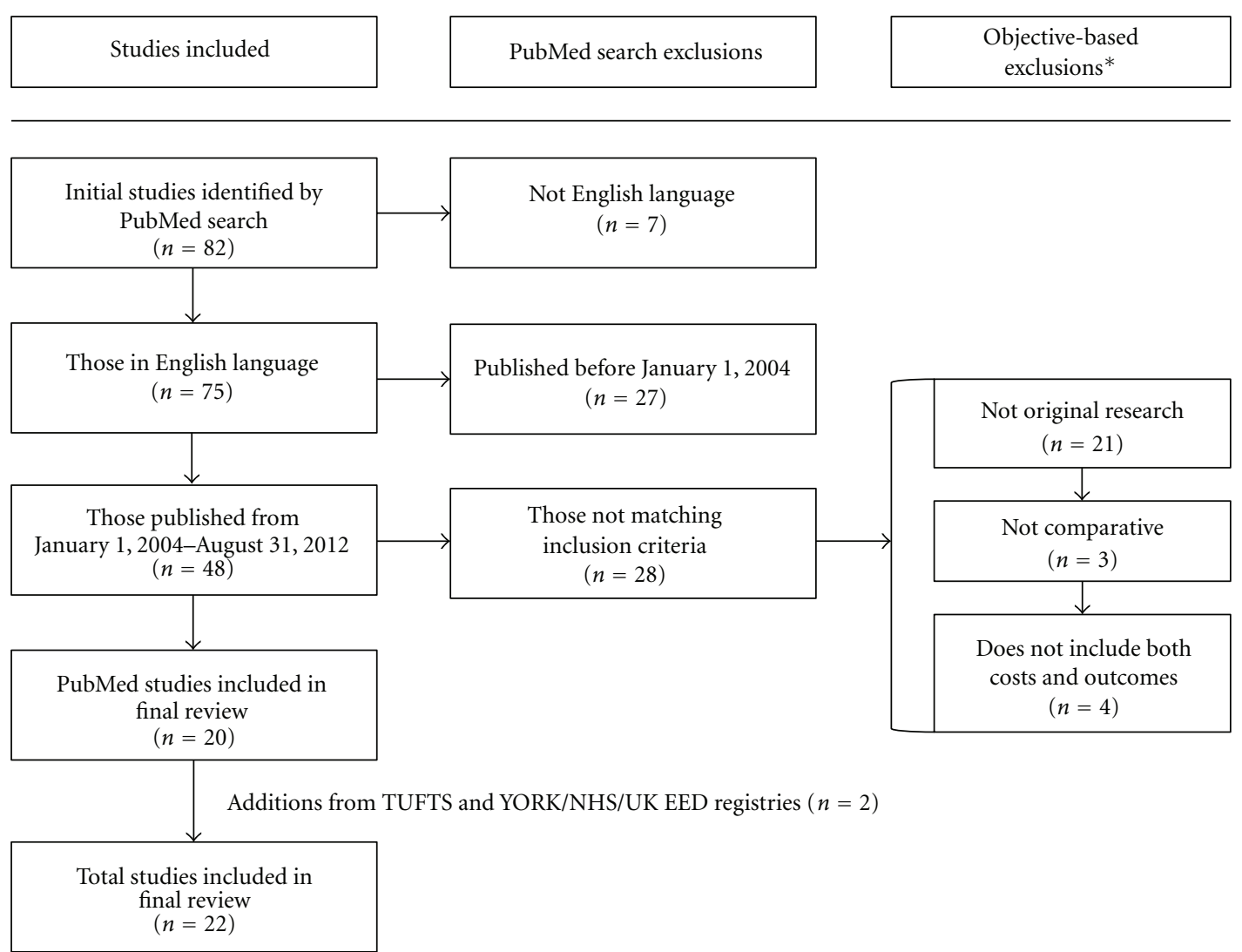

* Exclusion criteria are mutually exclusive, exhaustive, and adhere to the following hierarchy: not original research $>$ not comparative $>$ does not include both costs and outcomes.

FIGURE 1: Flowchart of systematic search methodology and yield.

However, the most germane consideration is whether the measure is relevant given the objectives of the decision maker concerned [28]. When there are multiple objectives of treatments or interventions, an array of differential achievements along each dimension may be presented for the alternative interventions. These data can then be used at the discretion of the decision maker to most appropriately address their unique situation [28]. This form of evaluation is referred to as cost-consequence analysis (CCA). Another form of CEA, cost-utility analysis (CUA), seeks to incorporate the weighted measures of all relevant outcomes in one measure based upon overall utility. The most common outcome measure used is the quality-adjusted life year (QALY), which estimates the alternative-specific survival and assigns utility weights ranging from zero (death) to one (perfect health) for each life year [30].

CEA studies follow a decision-analytic modeling (DAM) approach where currently available evidence concerning the effectiveness and costs of alternative healthcare interventions is synthesized in order to inform decision makers about the relative value of competing alternatives [31]. DAM helps decision makers to formulate as informed decisions as possible under conditions of cost and effectiveness uncertainty and resource scarcity. There are two main subbranches of DAM of interest for CEA of DMTs in MS: simulation models and patient-level trials. Simulation models can either be based upon patient cohorts or individual patient-level data. The most common type of cohort simulation model (CSM) in MS is the Markov model, where cohorts transition among multiple disease states via assigned probabilities calculated for specific time periods (cycles). Each disease state is assigned costs and outcomes in order to calculate outputs [28]. Patient-level trials are typically conducted within one randomized trial or within one observational study where both clinical and economic analyses are performed using the patient-level data.

2.2. Literature Search. We performed a systematic search in September of 2012 using MEDLINE (PubMed) querying for the MeSH term, "cost-benefit analysis," and the general search term, "multiple sclerosis." The MeSH term "costbenefit analysis" includes the following nested entry terms: cost-benefit, cost-effectiveness, and benefits and costs. There is no other MeSH term comprehensive enough to require elements of both costs and health outcomes. To control for the impact of including DMT generic and brand names in the query, we included (mitoxantrone OR interferon OR glatiramer acetate OR natalizumab OR fingolimod OR avonex OR betaseron OR extavia OR rebif OR novantrone OR copaxone OR gilenya OR tysabri) in addition to the initial 
search terms as part of an additional search for comparative purposes. We utilized PubMed search filters to limit the initial search to articles published in the English language from January 1, 2004 to August 31, 2012. In addition to the PubMed filters applied, we imposed a three-point exclusion criterion to the search results. The exclusion criteria were mutually exclusive, exhaustive, and hierarchical in the following manner (see Figure 1):

(1) not original research;

(2) not comparative;

(3) does not include both costs and outcomes.

If an article was excluded under the first criterion, it would not be further scrutinized for adherence to the second or third criteria, and if it adhered to the first but not to the second, it would not be further scrutinized for adherence to the third criterion. Those that reached this final exclusion criterion and adhered to all three criteria were included in the final systematic review database. To ensure the comprehensiveness of our database, we performed two additional searches using the same queries and criteria via the Tufts Medical Center Cost-Effectiveness Analysis Registry (TUFTS) and the National Health Service Economic Evaluation Database (NHSEED). Additional articles that were not found in the initial search and adhered to our three-point criterion were added to the final database.

2.3. Quality Assessment of Health Economic Analyses. We quantitatively evaluated the quality of the MS DMT costeffectiveness studies in a systematic and transparent manner through the use of the 16-item Quality of Health Economic Studies (QHES) validated instrument [32]. Both authors independently applied the QHES scoring instrument to each of the articles included in the final database and came to an agreement on all scores for each study. The final reconciled scores were reported. Total QHES scores range from 0 to 100, with 100 representing a perfect score. Four categories have been established to stratify the studies from lowest to highest quality: lowest quality category 1 (total score: 0-25), quality category 2 (total score: $25.1-50$ ), quality category 3 (total score: $50.1-75)$, and highest quality category 4 (75.1$100)$.

2.4. QHES Subdomains. We identified four main topics to focus our discussion of the composition and quality of the included cost-effectiveness studies: (i) model structure (QHES items 1, 2, 8, 12, and 13), (ii) model inputs (QHES items 3, 7, 9, 10, and 11), (iii) results/conclusions (QHES items 6, 11, and 15), and (iv) study integrity (QHES items 5, 13,14 , and 16) [32]. Item 11 is included in both the model inputs and results/conclusions subdomains. Appropriate choice of model structure is of paramount importance in determining the reach and applicability of the results and conclusions of CEA studies. In addition, transparency and justification of the model framework and its assumptions are crucial to enable an unbiased peer review process as well as in determining the usability and repeatability of the study and its findings [30]. Appropriate acknowledgment of the type of data abstraction employed and justification for the methodology allows users to replicate and validate the analysis [30]. It is important that the model inputs are appropriately matched to the model structure employed, specifically with respect to the audience it is meant to inform (study perspective) and the analytical time horizon employed, in order to properly advance the knowledge pool that decision makers draw from in developing protocols for the treatment of MS. The main results of CEA studies are presented via the ICER. This ratio describes the incremental cost of an intervention for each additional unit of benefit. This form of presenting CEA study results allows for a standardized interpretation of cost-effectiveness between comparators and is the cornerstone of quality studies in this field. A negative ICER indicates that one of the alternatives is more effective and less costly than the other and is considered to dominate the other. A positive ICER indicates that one alternative is both less costly and less effective or more costly and more effective. An intervention is considered costeffective if it dominates its comparator or if it is both more (or less) costly and effective, while falling below the decision maker's willingness-to-pay threshold [30]. The resulting ICER and conclusion of a CEA study are only useful if it is justified based upon the study results. The integrity of a study relies on an explicit transparency with respect to uncertainty, limitations, assumptions, and bias. Clearly describing uncertainty with respect to individual variables in isolation (oneway sensitivity analysis), as well as in conjunction (multiway sensitivity analysis, probabilistic sensitivity analysis, etc.), informs decision makers as to which inputs have the greatest impact on results and indicates areas for future research.

\section{Results}

3.1. Literature Search Results. Of the 82 total studies that met our initial MEDLINE search criteria [1, 3, 4, 23-25, 31, 33107], we included 20 in our review (Figure 1) [1, 33, 35, 38, $39,48,52,55,58,59,62,66,77,78,80,84,100,101,106$, 107]. With respect to the PubMed filter exclusions, seven of 80 studies were not published in English and 27 others were published before January 1, 2004. The most common reason for excluding studies matching our initial search queries and filters was not reporting original research (i.e. reviews, methods papers, letters, and editorials were excluded). 21 studies were excluded due to this primary objective-based exclusion $[3,23,25,31,34,43-45,49,53,60,67,69,72,75$, $86,87,91,97,99,105]$. Three other studies were deemed not comparative $[4,54,83]$ and an additional four studies did not include both costs and outcomes [36, 65, 71, 88]. We located two articles $[108,109]$ in the TUFTS and NHSEED databases that met all of our criteria, resulting in a final total of 22 articles included in our review.

3.2. Results by Model Type. Table 2 contains characteristics of each study, including details pertaining to the model type chosen. 20 of the 22 studies were simulation models: 19 of these 20 were cohort simulation models (CSM) [1, 33, 35, 39, $48,52,58,59,62,66,77,78,80,84,100,101,106-108]$ and 
one was a patient-level simulation [55]. Of the 19 CSM, 14 were Markov models [35, 48, 52, 58, 59, 62, 77, 78, 84, 100, 101, 106-108]. Within the Markov models employed, four studies utilized one-month transition periods [35, 48, 59, $84]$, two studies utilized three-month transition periods [62, 108], two used six-month transition periods [106, 107], and six studies utilized one-year transition periods $[52,58,77,78$, $100,101]$.

We identified two patient-level trial-based studies: one based on an open-labeled head-to-head clinical trial [109] and one using data from a retrospective multivariate cohort analysis [38].

3.3. Results by Geographic Region and Perspective. 12 of the 22 studies were based upon data from the USA [1, 33, 35, 38, 39, $48,55,77,80,84,100,106]$. Of these USA studies, seven were performed under a payer/health care system perspective [1, $33,38,39,55,80,100]$, four were from a societal perspective $[35,77,84,106]$, and one included both perspectives [48]. There were two studies based upon data from the UK, one of which was from a societal perspective [52] and one was from a payer perspective [101]. There were two studies based upon data from Italy, one of which was from the payer perspective [109] and one included both societal and payer perspectives [66]. Two studies were derived from Swedish data and were performed from a societal perspectives [62, 107]. One study was derived from Canadian data and was performed from both societal and payer perspectives [58]. One study was derived from French data and was performed from both societal and payer perspectives [108]. One study was derived from Balkan data and was performed from a societal perspective [59]. One study was derived from German data and was performed from a societal perspective [78]. A total of nine studies utilized a payer perspective in their base-case analysis $[1,33,38,39,55,80,100,101,109]$, nine studies utilized a societal perspective in their base-case analysis [35, $52,59,62,77,78,84,106,107]$, and four studies included both perspectives in their base-case analysis $[48,58,66,108]$.

3.4. Results by DMT Type. Of the included studies, 16 included an interferon product $[1,33,35,38,39,52,55,58$, $59,66,77,78,84,100,106,107], 12$ included glatiramer acetate $[1,33,35,38,39,48,52,59,77,78,84,100]$, six included natalizumab $[33,39,48,52,62,80]$, two included mitoxantrone [101, 109], and one included fingolimod [80]. The cost-effectiveness of each DMT varied greatly depending upon the study design, perspective, and treatment comparisons chosen. However, natalizumab was found to be cost-effective or dominant in all studies it appeared in [33, $39,48,52,62,80]$, with one exception being the lifetime cost-effectiveness study from a healthcare perspective by Earnshaw et al. [48]. Mitoxantrone was not compared to another DMT in either of the studies in which it was included $[101,109]$.

3.5. Results by Primary Outcome and Time Horizon. The most prominent primary effectiveness outcome chosen was QALYs, which was represented in 13 of the 22 studies
$[35,48,52,59,62,66,77,84,100,101,106-108]$. The next most prevalent primary effectiveness outcome was relapses avoided, which was represented in six studies [1, 33, 39, 55, $78,80]$. Two studies included relapse rate reduction as a primary outcome $[38,109]$. Reduction in EDSS score [109], cost to health plan [39], relapse free days gained [55], and quality-adjusted monosymptomatic life years (QAMLY) [58] were chosen as primary outcomes each in one study. The studies with QALYs as the primary outcome had time horizons that ranged from 10 years to lifetime. The studies with relapses avoided as the primary outcome had time horizons of either two or four years.

3.6. Quality Assessment of Health Economic Analyses Results. Table 1 presents the QHES scores for each cost-effectiveness study. No studies were assigned quality category 1 , four studies were assigned quality category 2 [33, 38, 59, 109], three studies were assigned quality category $3[58,66,108]$, and 15 studies were assigned the highest quality category $4[1,35$, $39,48,52,55,62,77,78,80,84,100,101,106,107]$.

\subsection{QHES Subdomain Results}

3.7.1. Model Structure. In 21 of the 22 studies, the objective was "presented in a clear, specific, and measurable manner" (QHES item 1) $[1,35,38,39,48,52,55,58,59,62,66$, $77,78,80,84,100,101,106-109] .12$ studies appropriately stated and justified the perspective employed (QHES item 2) $[1,39,52,55,78,80,84,100,101,106-108] .16$ studies justified the time horizon chosen as appropriate to capture all important and relevant outcomes (QHES item 8) [1, 35, 39, $48,52,55,59,62,66,78,80,84,100,101,106,107]$. In 14 studies the economic model, methods, and analysis were transparent and repeatable (QHES item 12) [1, 35, 48, 52, $58,62,77,78,84,100,101,106-108] .14$ studies properly justified the model chosen (QHES item 13) [1, 35, 39, 48, 52, $55,62,77,80,100,101,106-108]$.

3.7.2. Model Inputs: Cost and Effectiveness Measures. Inputs were drawn from the best available source in 20 of the 22 studies (QHES item 3) [1, 33, 35, 38, 39, 48, 52, 55, 58, 62, $66,77,78,80,84,100,101,106-108]$. The data abstraction methodology was adequately stated and repeatable in 20 studies (QHES item 7) [1, 35, 39, 48, 52, 55, 58, 59, 62, 66, $77,78,80,84,100,101,106-109]$. The estimation of costs was appropriate and repeatable in 20 studies (QHES item 9) $[1,33,35,39,48,52,55,58,59,62,66,77,78,80,84,100,101$, 106-108]. In 16 studies the primary outcome measure was clearly stated and negative outcomes were included, or justification was given for their omission from the analysis (QHES item 10) [1, 35, 39, 48, 52, 55, 58, 66, 78, 80, 84, 100, $101,106,107,109]$. 19 studies chose valid primary outcome measures and justified them adequately (QHES item 11) $[1,33,35,38,39,48,52,55,62,66,77,78,80,84,100,101$, $106,107,109]$.

3.7.3. Results and Conclusions. 20 of the 22 studies performed an incremental analysis for costs and outcomes between 


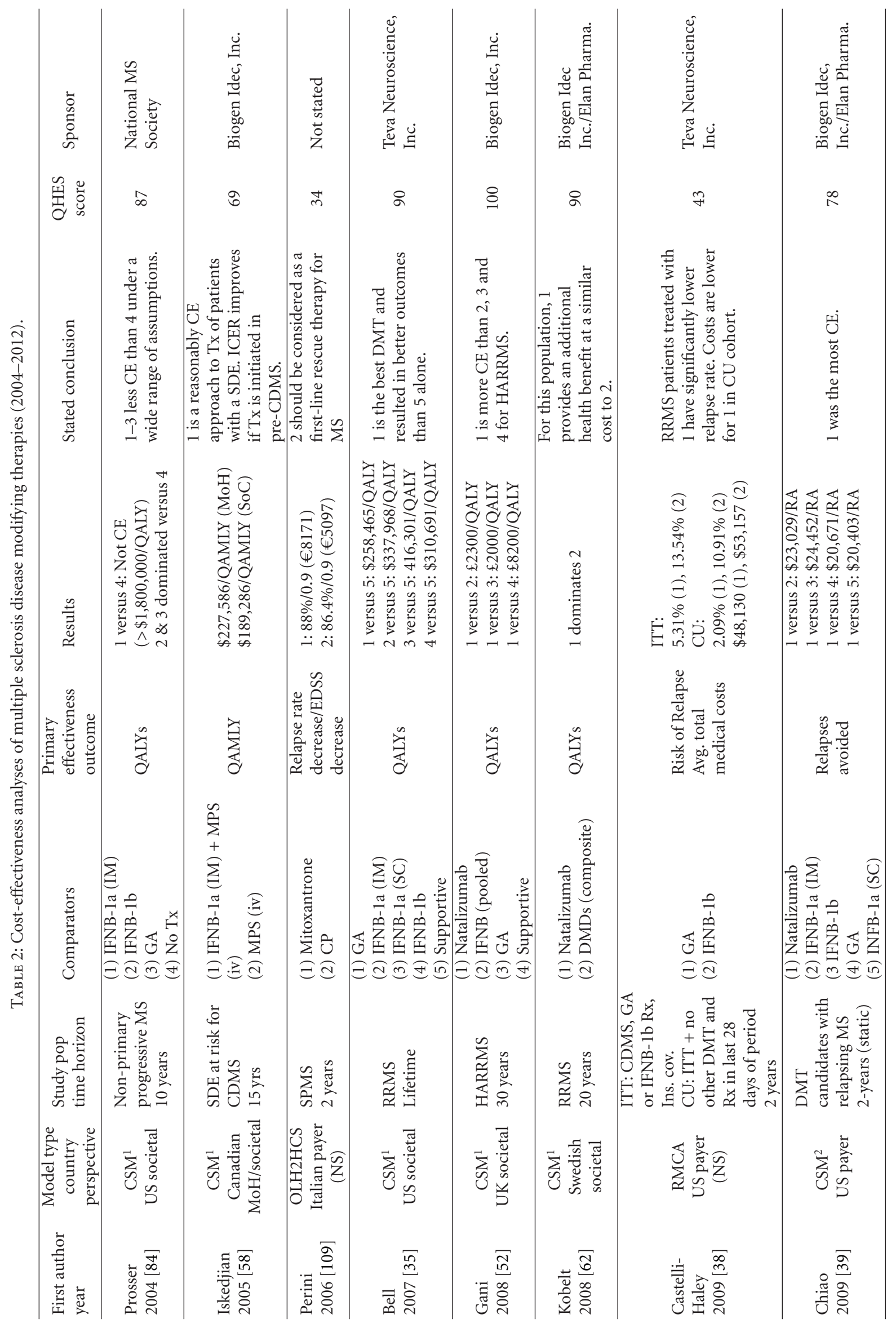




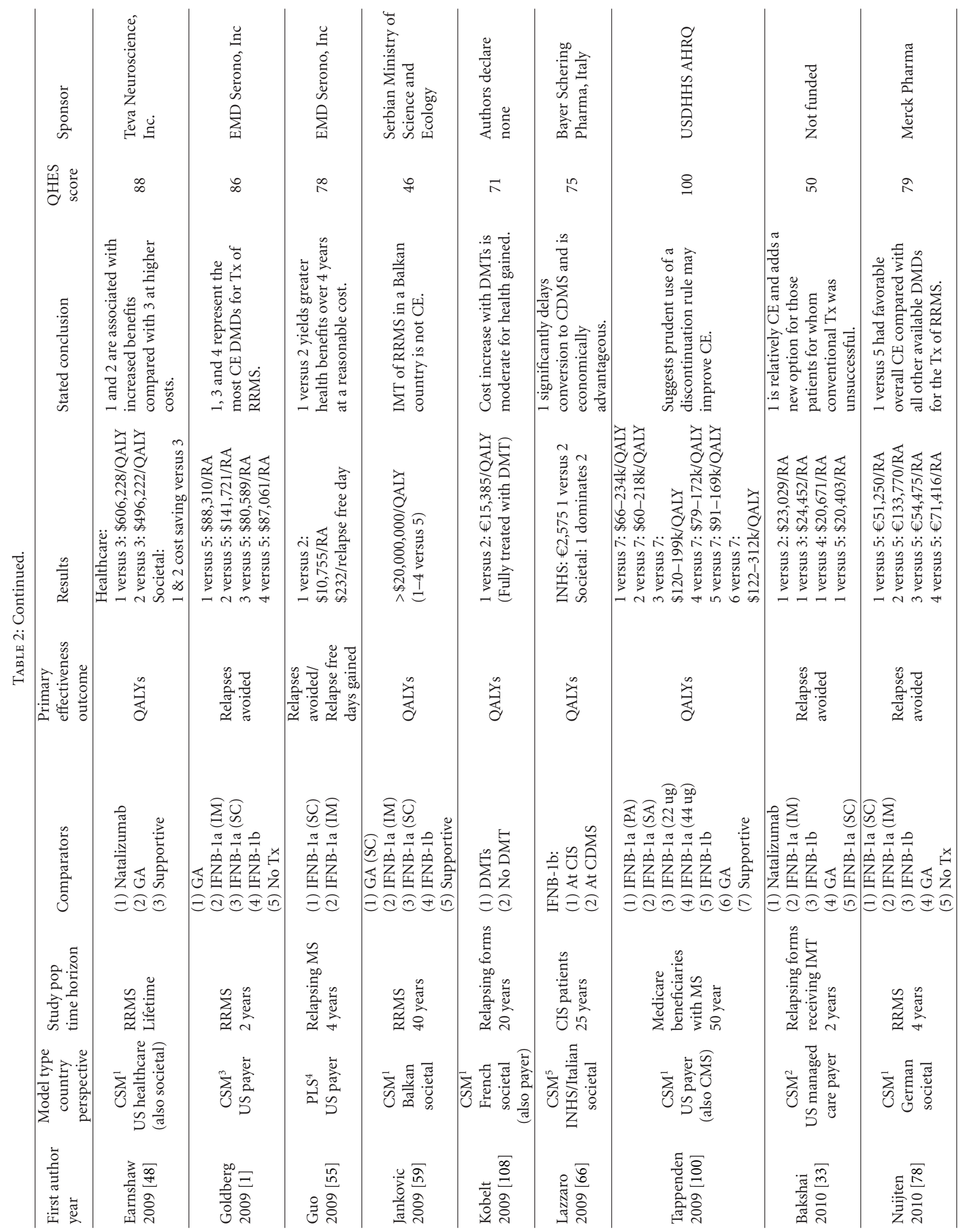




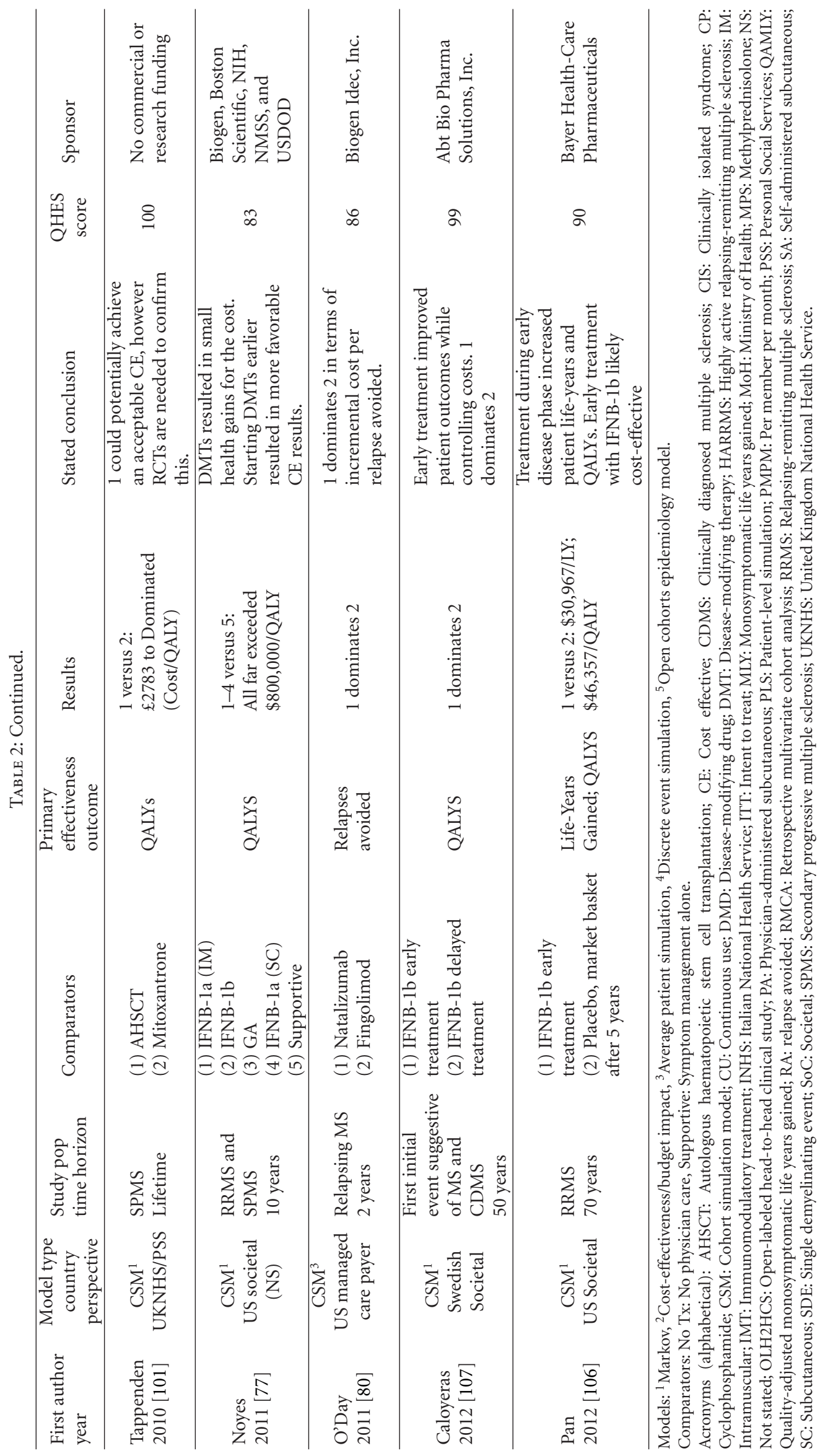


alternatives (QHES item 6) [1, 33, 35, 39, 48, 52, 55, 58, 59, $62,66,77,78,80,84,100,101,106-108] .15$ studies provided stated conclusions that were justified and based upon study results (QHES item 15) [33, 35, 38, 52, 58, 62, 66, 77, 80, 84, 100, 101, 106-108].

3.7.4. Study Integrity: Uncertainty, Limitations, Assumptions, Bias, and Funding. 20 of the 22 studies addressed uncertainty (QHES item 5) [1, 33, 35, 38, 39, 48, 52, 55, 58, 59, 62, $66,77,78,80,84,100,101,107,109] .14$ studies explicitly stated and justified the assumptions and limitations of the chosen model (QHES item 13) [1, 35, 39, 48, 52, 55, 62, 77, $80,100,101,106-108]$. Nine studies explicitly discussed the magnitude and direction of potential biases (QHES item 14) $[48,52,62,77,100,101,106-108] .21$ studies provided a statement disclosing the source of funding for the study (QHES item 16) $[1,33,35,38,39,48,52,55,58,59,62,66$, $77,78,80,84,100,101,106-108]$.

\section{Discussion}

Clinical guidelines for the treatment of MS with DMTs remain underdeveloped and lacking in comprehensive understanding and consensus regarding what DMT should be used for what type of MS patient. The American Academy of Neurology (AAN) and the MS Council for Clinical Practice Guidelines have not published comprehensive guidelines including all current DMTs for the US since 2002 (guidelines were reaffirmed in 2008) [110]. In the UK, the National Institute for Clinical Excellence (NICE) relies on guidelines for the treatment of MS that were published in 2003 [111]. In parallel to the development of current and accurate clinical guidelines is the need for quality and consistency in CEA of DMTs for MS. We compare our systematic review findings with past recommendations and highlight areas of progress and those in need of further development.

There was a preponderance of simulation models within our included studies. Simulation modeling allows for the projection of short-term data to reflect the chronic nature of MS. The use of simulation models in lieu of long-term DMT studies indicates progress in the field and is consistent with previous recommendations regarding the use of simulation modeling when long-term cost and outcome studies are lacking. The large majority of these simulation models employed a Markov structure, which allows for long-term analysis, up to lifetime in scope. Markov modeling also allows for transition between disease states for cohorts of patients, which reflects the natural disease progression within MS. This further indicates a positive trajectory in the costeffectiveness evaluation of DMTs for MS.

The past recommendations supporting use of the longterm time horizons and societal perspectives harmonize well with the use of QALYs as the primary outcome measure, as was suggested by Kobelt [26]. The majority of studies utilized long-term time horizons. More than half of the studies included a societal perspective in their base-case analysis and utilized QALYs as their primary outcome measure indicating progress, albeit with room for improvement in adhering to past recommendations. However, there were a significant number of studies that utilized a two- or four-year time horizon, payer perspective, and relapses avoided as their primary outcome measures. The short-term time horizon studies provide evidence for example to USA insurers who may have 2- to 4-year average insured time horizons for their populations. Past recommendations can be advanced by suggesting that CEA studies on DMTs for MS should primarily adopt a long-term time horizon, societal perspective, and QALYs as the fundamental recommendation, with the option to supplement the study with an added analysis using short-term time horizons, payers perspectives, and clinical effectiveness (or safety) measures such as relapses avoided. A cost-consequences approach would allow for more than one effectiveness (or safety) measure to be compared across DMTs and should be considered depending upon the decision makers' needs.

The assumptions employed in the simulation models were diverse and inconsistent between studies indicating much room for improvement in conforming to past recommendations with respect to consistency in modeling. This diversity is a factor in the wide range of cost-effectiveness estimates for the same DMTs across studies. The generally poor performance of studies in explicitly stating and properly justifying model assumptions, discussing the magnitude and direction of potential biases, employing transparent and repeatable models/methods, and justifying their choice of model (QHES items 12, 13, and 14) further indicates a lack of adherence to proper guidelines within the field for consistent and appropriate modeling methodology. We call for more efforts in the standardization of cost-effectiveness studies within MS and for studies to provide rationale for why the design and assumptions may differ from previous costeffectiveness studies.

The lack of head-to-head randomized controlled trials (RCTs) between DMTs and absence of long-term observational data serve to drive the need for cost-effectiveness studies to employ multiple assumptions since the appropriate long-term comparative data do not exist. The currently available RCT data are generally specific to individual DMTs and are based upon different patient populations and different study characteristics such as treatment adherence, dropout rates, and adverse outcomes. Therefore, the results of individual studies are contingent upon which RCT data are employed, along with the assumptions included in the model. This heterogeneity makes comparing the results across studies difficult. More comparative head-to-head RCTs among DMTs and prospective observational studies are needed and will generate less heterogeneity in costeffectiveness model structures and inputs yielding less uncertainty in cost-effectiveness results. It is important that proper guidelines for cost-effectiveness modeling [112] are utilized by researchers to maintain consistency and comparability within the field. Progress of this order is necessary to satisfy the recommendation that standard modeling is employed across studies, which we found to still be largely unfulfilled in recent studies.

Finally, there was a lack of cost-effectiveness studies on fingolimod. There is a need for studies including fingolimod 
as a comparator, specifically coupled with traditional DMTs (interferons and glatiramer acetate) to determine this new treatment's value as a therapy option in MS. Given the high annual treatment cost of fingolimod, the cost-effectiveness evidence as compared to other DMTs becomes even more important. There were no studies that included all approved DMTs as comparators indicating a need for a comprehensive study including all appropriate DMTs for particular MS patient populations. A comprehensive cost-effectiveness study of this nature would help to alleviate the problems of comparing cost-effectiveness across different studies that employ different methodologies and study assumptions.

\section{Conclusion}

The cost-effectiveness body of evidence of DMTs for the treatment of MS has shown progress in responding to the recommendation of past reviews, while there remains room for improvement in many areas. The area of greatest advancement is in the use of simulation models that represent the chronic nature of the disease. This appears to be the dominant trend in current studies. To a lesser degree, the field has shown progress in adhering to the recommendation that long-term time horizons, societal perspectives, and QALYs are utilized, albeit with room for further improvement. We recommend that studies utilize lifetime horizons, societal perspectives, and QALYs as the primary standards in CEA studies of DMTs for MS, with the option to supplement the base-case analysis by including shortterm horizons, payer perspectives, and a cost-consequences approach. This recommendation conforms to the suggestions of past reviewers, while adding the ability for individual studies to inform multiple decision makers. There is a great need for improvement in the standardization of modeling procedures and data inputs. We recommend that modeling and input assumption standards are developed within MS cost-effectiveness studies to aid comparability across future studies. The recommendation to perform head-to-head RCTs between DMTs and collect long-term prospective observational data would improve study consistency in the future. Finally, we recommend that comprehensive studies comparing all approved DMTs in concert are performed to help control for the inconsistencies between studies and provide meaningful results for decision makers.

\section{Acknowledgments}

This research was funded in part by the Agency for Healthcare Research and Quality as part of the Colorado Comparative Effectiveness Research K12 Training Program: K12 HS019464. No other funding source was directly tied to this study.

\section{References}

[1] L. D. Goldberg, N. C. Edwards, C. Fincher, Q. V. Doan, A. Al-Sabbagh, and D. M. Meletiche, "Comparing the costeffectiveness of disease-modifying drugs for the first-line treatment of relapsing-remitting multiple sclerosis," Journal of Managed Care Pharmacy, vol. 15, no. 7, pp. 543-555, 2009.

[2] H. L. Zwibel and J. Smrtka, "Improving quality of life in multiple sclerosis: an unmet need," The American Journal of Managed Care, vol. 17, supplement 5, pp. S139-S145, 2011.

[3] C. J. Phillips, "The cost of multiple sclerosis and the cost effectiveness of disease-modifying agents in its treatment," CNS Drugs, vol. 18, no. 9, pp. 561-574, 2004.

[4] S. Olofsson, A. Wickstrom, A. Hager Glenngard, U. Persson, and A. Svenningsson, "Effect of treatment with natalizumab on ability to work in people with multiple sclerosis: productivity gain based on direct measurement of work capacity before and after 1 year of treatment," BioDrugs, vol. 25, no. 5, pp. 299-306, 2011.

[5] Multiple Sclerosis Association of America, Treatments for Multiple Sclerosis, 2012, http://www.msassociation.org/ about_multiple_sclerosis/treating/.

[6] J. A. Schafer, B. W. Gunderson, and P. P. Gleason, "Price increases and new drugs drive increased expenditures for multiple sclerosis," Journal of Managed Care Pharmacy, vol. 16, no. 9, pp. 713-717, 2010.

[7] G. Kobelt, P. Lindgren, A. Smala et al., "Costs and quality of life in multiple sclerosis. An observational study in Germany," HEPAC Health Economics in Prevention and Care, vol. 2, no. 2, pp. 60-68, 2001.

[8] G. Kobelt, P. Lindgren, D. Parkin et al., "Costs and quality of life in multiple sclerosis: a cross-sectional observational study in the United Kingdom," EFI Research Reports, Stockholm, Sweden, 2000.

[9] F. Henriksson, S. Fredrikson, T. Masterman, and B. Jönsson, "Costs, quality of life and disease severity in multiple sclerosis: a cross-sectional study in Sweden," European Journal of Neurology, vol. 8, no. 1, pp. 27-35, 2001.

[10] D. T. Grima, G. W. Torrance, G. Francis, G. Rice, A. J. Rosner, and L. Lafortune, "Cost and health related quality of life consequences of multiple sclerosis," Multiple Sclerosis, vol. 6, no. 2, pp. 91-98, 2000.

[11] G. Kobelt, J. Berg, D. Atherly, and O. Hadjimichael, "Costs and quality of life in multiple sclerosis: a cross-sectional study in the United States," Neurology, vol. 66, no. 11, pp. 16961702, 2006.

[12] P. Russo, A. Capone, A. Paolillo et al., "Cost-analysis of relapsing-remitting multiple sclerosis in Italy after the introduction of new disease-modifying agents," Clinical Drug Investigation, vol. 24, no. 7, pp. 409-420, 2004.

[13] P. D. R. Staff Thomson, Red Book: Pharmacy's Fundamental Reference, Thomson Healthcare, 114th edition, 2010.

[14] Gilenya (fingolimod) [package insert], East Hanover, NJ, USA, Novartis, 2010.

[15] B. H. Pharmaceuticals, "Betaseron Prescribing Information," 2012, http://berlex.bayerhealthcare.com/html/products/pi/ Betaseron_PI.pdf.

[16] I. Biogen, “Avonex Prescribing Information,” 2012, http://www .avonex.com/pdfs/guides/Avonex_Prescribing_Information .pdf.

[17] I. Teva Neuroscience, "Copaxone Prescribing Information," 2010, http://www.sharedsolutions.com/redirect/PrescribingInformation.pdf.

[18] National Center for Biotechnology Information, "Novantrone Black Box Warning," 2010, http://www.ncbi.nlm.nih .gov/books/NBK50576/.

[19] E. M. D. Serono I, "Rebif Prescribing Information," 2012, http://www.emdserono.com/cmg.emdserono_us/en/images/ rebif_tcm115_19765.pdf. 
[20] I. Elan Pharmaceuticals, "Tysabri Prescriber Information," 2012, http://www.tysabri.com/pdfs/I61061-13_PI.pdf.

[21] N. P. Corporation, "Extavia Important Product Information (Prescribing Information),” 2012, http://www.pharma.us.novartis.com/product/pi/pdf/extavia.pdf.

[22] N. P. Coporation, "Gilenya Prescribing Information," 2012, http://www.pharma.us.novartis.com/product/pi/pdf/gilenya .pdf.

[23] M. P. Amato, "Pharmacoeconomic considerations of multiple sclerosis therapy with the new disease-modifying agents," Expert Opinion on Pharmacotherapy, vol. 5, no. 10, pp. 21152126, 2004.

[24] P. Flachenecker and P. Rieckmann, "Early intervention in multiple sclerosis: better outcomes for patients and society?" Drugs, vol. 63, no. 15, pp. 1525-1533, 2003.

[25] P. Flachenecker and P. Rieckmann, "Health outcomes in multiple sclerosis," Current Opinion in Neurology, vol. 17, no. 3, pp. 257-261, 2004.

[26] G. Kobelt, "Health economic issues in MS," International MS Journal / MS Forum, vol. 13, no. 1, pp. 16-17, 2006.

[27] P. Rieckmann, "Socio-economic aspects of neuroimmunological diseases," Journal of Neurology, vol. 253, no. 5, pp. V 87-V 90, 2006.

[28] F. Drummond Michael SMJ, W. Torrance George, J. O.'Brien Bernie, and L. Stoddart Greg, Methods for the Economic Evaluation of Health Care Programmes, Oxford University Press, New York, NY, USA, 3rd edition, 2005.

[29] F. Sloan, Valuing Health Care: Costs, Benefits, and Effectiveness of Pharmaceuticals and Other Medical Technologies, Cambridge University Press, Cambridge, UK, USA, 1996.

[30] J. D. Campbell, D. E. Spackman, and S. D. Sullivan, "Health economics of asthma: assessing the value of asthma interventions," Allergy, vol. 63, no. 12, pp. 1581-1592, 2008.

[31] Z. Philips, L. Bojke, M. Sculpher, K. Claxton, and S. Golder, "Good practice guidelines for decision-analytic modelling in health technology assessment: a review and consolidation of quality assessment," PharmacoEconomics, vol. 24, no. 4, pp. 355-371, 2006.

[32] C. F. Chiou, J. W. Hay, J. F. Wallace et al., "Development and validation of a grading system for the quality of cost-effectiveness studies," Medical Care, vol. 41, no. 1, pp. 32-44, 2003.

[33] J. Bakhshai, R. Bleu-Lainé, M. Jung et al., "The cost effectiveness and budget impact of natalizumab for formulary inclusion," Journal of Medical Economics, vol. 13, no. 1, pp. 63-69, 2010.

[34] R. V. Becker III and C. Dembek, "Effects of cohort selection on the results of cost-effectiveness analysis of disease-modifying drugs for relapsing-remitting multiple sclerosis," Journal of Managed Care Pharmacy, vol. 17, no. 5, pp. 377-381, 2011.

[35] C. Bell, J. Graham, S. Earnshaw, M. Oleen-Burkey, J. Castelli-Haley, and K. Johnson, "Cost-effectiveness of four immunomodulatory therapies for relapsing-remitting multiple sclerosis: a Markov model based on long-term clinical data," Journal of Managed Care Pharmacy, vol. 13, no. 3, pp. 245261, 2007.

[36] M. Boggild, J. Palace, P. Barton et al., "Multiple sclerosis risk sharing scheme: two year results of clinical cohort study with historical comparator," British Medical Journal, vol. 339, p. b4677, 2009.

[37] M. G. Brown, T. Jock Murray, I. S. Sketris et al., "Cost-effectiveness of interferon beta-1b in slowing multiple sclerosis disability progression: first estimates," International Journal of Technology Assessment in Health Care, vol. 16, no. 3, pp. 751-767, 2000.

[38] J. Castelli-Haley, M. A. Oleen-Burkey, M. J. Lage, and K. P. Johnson, "Glatiramer acetate and interferon beta-1b: a study of outcomes among patients with multiple sclerosis," Advances in Therapy, vol. 26, no. 5, pp. 552-562, 2009.

[39] E. Chiao and K. Meyer, "Cost effectiveness and budget impact of natalizumab in patients with relapsing multiple sclerosis," Current Medical Research and Opinion, vol. 25, no. 6, pp. 1445-1454, 2009.

[40] J. Chilcott, C. McCabe, P. Tappenden et al., "Modelling the cost effectiveness of interferon beta and glatiramer acetate in the management of multiple sclerosis," British Medical Journal, vol. 326, no. 7388, pp. 522-525, 2003.

[41] A. Clegg and J. Bryant, "Immunomodulatory drugs for multiple sclerosis: a systematic review of clinical and cost effectiveness," Expert Opinion on Pharmacotherapy, vol. 2, no. 4, pp. 623-639, 2001.

[42] A. Clegg, J. Bryant, and R. Milne, "Disease-modifying drugs for multiple sclerosis: a rapid and systematic review," Health Technology Assessment, vol. 4, no. 9, pp. 1-101, 2000.

[43] A. Coles, “The fragile benefit of BENEFIT," Lancet Neurology, vol. 6, no. 9, pp. 753-754, 2007.

[44] A. Compston, "Commentary: scheme has benefited patients," British Medical Journal, vol. 340, p. c2707, 2010.

[45] F. R. Curtiss, "Pharmacoeconomic modeling of drug therapies for multiple sclerosis-are we building houses on sand?" Journal of Managed Care Pharmacy, vol. 13, no. 3, pp. 287289, 2007.

[46] B. Detournay, "The value of economic modeling studies in the evaluation of treatment strategies for multiple sclerosis," Value in Health, vol. 5, no. 1, pp. 1-2, 2002.

[47] N. Devlin, J. Appleby, and D. Parkin, "Patients' views of explicit rationing: what are the implications for health service decision-making?" Journal of Health Services Research and Policy, vol. 8, no. 3, pp. 183-186, 2003.

[48] S. R. Earnshaw, J. Graham, M. Oleen-Burkey, J. CastelliHaley, and K. Johnson, "Cost effectiveness of glatiramer acetate and natalizumab in relapsing-remitting multiple sclerosis," Applied Health Economics and Health Policy, vol. 7, no. 2, pp. 91-108, 2009.

[49] G. C. Ebers, "Commentary: outcome measures were flawed," British Medical Journal, vol. 340, p. c2693, 2010.

[50] S. J. Ellis, “Bad decision NICE,” The Lancet, vol. 359, no. 9304, p. 447, 2002.

[51] R. B. Forbes, A. Lees, N. Waugh, and R. J. Swingler, "Population based cost utility study of interferon beta-1b in secondary progressive multiple sclerosis," British Medical Journal, vol. 319, no. 7224, pp. 1529-1533, 1999.

[52] R. Gani, G. Giovannoni, D. Bates, B. Kemball, S. Hughes, and J. Kerrigan, "Cost-effectiveness analyses of natalizumab (Tysabriß) compared with other disease-modifying therapies for people with highly active relapsing-remitting multiple sclerosis in the UK," PharmacoEconomics, vol. 26, no. 7, pp. 617-627, 2008.

[53] R. E. Gonsette, "A comparison of the benefits of mitoxantrone and other recent therapeutic approaches in multiple sclerosis," Expert Opinion on Pharmacotherapy, vol. 5, no. 4, pp. 747-765, 2004.

[54] J. Greenhalgh, H. Ford, A. F. Long, and K. Hurst, "The MS Symptom and Impact Diary (MSSID): psychometric evaluation of a new instrument to measure the day to day impact 
of multiple sclerosis," Journal of Neurology, Neurosurgery and Psychiatry, vol. 75, no. 4, pp. 577-582, 2004.

[55] S. Guo, D. Bozkaya, A. Ward et al., "Treating relapsing multiple sclerosis with subcutaneous versus intramuscular interferon-beta-1a: modelling the clinical and economic implications," PharmacoEconomics, vol. 27, no. 1, pp. 39-53, 2009.

[56] J. G. Heller, "Will public health survive QALYs?" Canadian Journal of Clinical Pharmacology, vol. 9, no. 1, pp. 5-6, 2002.

[57] R. M. Herndon and L. Jacobs, "Interferons should be used to treat most patients with MS," Archives of Neurology, vol. 55, no. 12, pp. 1581-1583, 1998.

[58] M. Iskedjian, J. H. Walker, T. Gray, C. Vicente, T. R. Einarson, and A. Gehshan, "Economic evaluation of Avonex (interferon beta-la) in patients following a single demyelinating event," Multiple Sclerosis, vol. 11, no. 5, pp. 542-551, 2005.

[59] S. M. Jankovic, M. Kostic, M. Radosavljevic et al., "Costeffectiveness of four immunomodulatory therapies for relapsing-remitting multiple sclerosis: a Markov model based on data a Balkan country in socioeconomic transition," Vojnosanitetski Pregled, vol. 66, no. 7, pp. 556-562, 2009.

[60] K. P. Johnson and D. L. Due, "Benefits of glatiramer acetate in the treatment of relapsing-remitting multiple sclerosis," Expert Review of Pharmacoeconomics and Outcomes Research, vol. 9, no. 3, pp. 205-214, 2009.

[61] M. Kendrick and K. I. Johnson, "Long term treatment of multiple sclerosis with interferon- $\beta$ may be cost effective," PharmacoEconomics, vol. 18, no. 1, pp. 45-53, 2000.

[62] G. Kobelt, J. Berg, P. Lindgren, B. Jonsson, L. Stawiarz, and J. Hillert, "Modeling the cost-effectiveness of a new treatment for MS (natalizumab) compared with current standard practice in Sweden," Multiple Sclerosis, vol. 14, no. 5, pp. 679-690, 2008.

[63] G. Kobelt, L. Jönsson, F. Henriksson, S. Fredrikson, and B. Jönsson, "Cost-utility analysis of interferon beta-1b in secondary progressive multiple sclerosis," International Journal of Technology Assessment in Health Care, vol. 16, no. 3, pp. 768-780, 2000.

[64] G. Kobelt, L. Jönsson, C. Miltenburger, and B. Jönsson, "Cost-utility analysis of interferon beta-1b in secondary progressive multiple sclerosis using natural history disease data," International Journal of Technology Assessment in Health Care, vol. 18, no. 1, pp. 127-138, 2002.

[65] M. Lapé Nixon, J. Matud, C. Yeh, and H. E. Prince, "Evaluation of a multiplex bead-based screening assay for detection of binding antibodies to interferon-beta," Journal of Neuroimmunology, vol. 210, no. 1-2, pp. 104-107, 2009.

[66] C. Lazzaro, C. Bianchi, L. Peracino, P. Zacchetti, and A. Uccelli, "Economic evaluation of treating clinically isolated syndrome and subsequent multiple sclerosis with interferon $\beta$-1b," Neurological Sciences, vol. 30, no. 1, pp. 21-31, 2009.

[67] R. K. Lie, "Research ethics and evidence based medicine," Journal of Medical Ethics, vol. 30, no. 2, pp. 122-125, 2004.

[68] R. MacDonald, "Cost effectiveness of multiple sclerosis drugs remains unknown," British Medical Journal, vol. 321, no. 7258, p. 399, 2000.

[69] C. McCabe, J. Chilcott, K. Claxton et al., "Continuing the multiple sclerosis risk sharing scheme is unjustified," British Medical Journal, vol. 340, p. c1786, 2010.

[70] L. McKee, "Interferon beta produces only small benefits in multiple sclerosis," British Medical Journal, vol. 316, no. 7142, p. 1410, 1998.

[71] P. McNamee, "What difference does it make? The calculation of QALY gains from health profiles using patient and general population values," Health Policy, vol. 84, no. 2-3, pp. 321331, 2007.

[72] H. McNaughton, N. Kayes, and K. McPherson, "Interferon beta, PHARMAC, and political directives: in the best interests of people with multiple sclerosis?" New Zealand Medical Journal, vol. 119, no. 1232, p. U1939, 2006.

[73] F. Medina-Redondo, J. Herrera-Carranza, C. Sanabria et al., "The efficiency and cost-utility ratio of interferon beta in the treatment of multiple sclerosis in Andalusia," Revista de Neurologia, vol. 39, no. 1, pp. 1-6, 2004.

[74] C. M. Meyer, R. Phipps, D. Cooper, and A. Wright, "Pharmacy benefit forecast for a new interferon Beta-1a for the treatment of multiple sclerosis: development of a first-line decision tool for pharmacy-budget planning using administrative claims data," Journal of Managed Care Pharmacy, vol. 9, no. 2, pp. 168-174, 2003.

[75] D. Murdoch and K. A. Lyseng-Williamson, "Subcutaneous recombinant interferon- $\beta$-1a (Rebif): a review of its use in relapsing-remitting multiple sclerosis," Drugs, vol. 65, no. 9, pp. 1295-1312, 2005.

[76] J. C. Napier, R. Francis, and G. Wright, "Shared scheme for assessing drugs for multiple sclerosis: cost effective provision of effective treatments for multiple sclerosis," British Medical Journal, vol. 326, no. 7400, p. 1212, 2003.

[77] K. Noyes, A. Bajorska, A. Chappel et al., "Cost-effectiveness of disease-modifying therapy for multiple sclerosis: a population-based study," Neurology, vol. 77, no. 4, pp. 355363, 2011.

[78] M. Nuijten and T. Mittendorf, "A health-economic evaluation of disease-modifying drugs for the treatment of relapsing-remitting multiple sclerosis from the German societal perspective," Clinical Therapeutics, vol. 32, no. 4, pp. 717728, 2010.

[79] M. J. C. Nuijten and J. Hutton, "Cost-effectiveness analysis of interferon beta in multiple sclerosis: a markov process analysis," Value in Health, vol. 5, no. 1, pp. 44-54, 2002.

[80] K. O’ Day, K. Meyer, R. M. Miller, S. Agarwal, and M. Franklin, "Cost-effectiveness of natalizumab versus fingolimod for the treatment of relapsing multiple sclerosis," Journal of Medical Economics, vol. 14, no. 5, pp. 617-627, 2011.

[81] D. Parkin, A. Jacoby, P. McNamee, P. Miller, S. Thomas, and D. Bates, "Treatment of multiple sclerosis with interferon $\beta$ : an appraisal of cost-effectiveness and quality of life," Journal of Neurology Neurosurgery and Psychiatry, vol. 68, no. 2, pp. 144-149, 2000.

[82] D. Parkin, P. McNamee, A. Jacoby, P. Miller, S. Thomas, and D. Bates, "A cost-utility analysis of interferon beta for multiple sclerosis," Health Technology Assessment, vol. 2, no. 4, pp. 3-54, 1998.

[83] M. Pickin, C. L. Cooper, T. Chater et al., "The multiple sclerosis risk sharing scheme monitoring study-early results and lessons for the future," BMC Neurology, vol. 9, article 1, 2009.

[84] L. A. Prosser, K. M. Kuntz, A. Bar-Or, and M. C. Weinstein, "Cost-effectiveness of interferon beta- $1 \mathrm{a}$, interferon beta-1b, and glatiramer acetate in newly diagnosed non-primary progressive multiple sclerosis," Value in Health, vol. 7, no. 5, pp. 554-568, 2004.

[85] W. Pryse-Phillips, "Newer long-term treatments for multiple sclerosis," Clinical Neurology and Neurosurgery, vol. 104, no. 3, pp. 265-271, 2002.

[86] J. Raftery, "Multiple sclerosis risk sharing scheme: a costly failure," BMBritish Medical Journal, vol. 340, p. c1672, 2010. 
[87] N. Rawson, "Response to McNaughton and colleagues regarding their article-interferon beta, PHARMAC, and political directives: in the best interests of people with multiple sclerosis?" The New Zealand Medical Journal, vol. 119, no. 1235, p. U2018, 2006.

[88] M. W. Reynolds, R. Stephen, C. Seaman, and K. Rajagopalan, "Healthcare resource utilization following switch or discontinuation in multiple sclerosis patients on disease modifying drugs," Journal of Medical Economics, vol. 13, no. 1, pp. 9098, 2010.

[89] S. J. Rich and C. Meyer, "Shortcomings in pharmacy benefit forecasting-interferon beta products," Journal of Managed Care Pharmacy, vol. 9, no. 4, pp. 373-374, 2003.

[90] R. G. Richards, "Interferon beta in multiple sclerosis," British Medical Journal, vol. 313, no. 7066, p. 1159, 1996.

[91] R. G. Richards, "MS risk sharing scheme. Some clarification needed," British Medical Journal, vol. 341, p. c3589, 2010.

[92] P. Rieckmann, "Early multiple sclerosis therapy in the effects of public health economics," Medizinische Klinik, vol. 96, supplement 1, pp. 17-21, 2001.

[93] P. Rieckmann and K. V. Toyka, "Immunomodulatory staged therapy of multiple sclerosis. New aspects and practical applications, March 2002," Nervenarzt, vol. 73, no. 6, pp. 556-563, 2002.

[94] C. Rubio-Terrés, I. Arístegui Ruiz, F. Medina Redondo, and G. Izquierdo Ayuso, "Cost-utility analysis of multiple sclerosis treatment with glatiramer acetate or interferon beta in Spain," Farmacia Hospitalaria, vol. 27, no. 3, pp. 159-165, 2003.

[95] C. Rubio-Terres and A. Dominguez-Gil Hurle, "Cost-utility analysis of relapsing-remitting multiple sclerosis treatment with azathioprine or interferon beta in Spain," Revista de Neurología, vol. 40, no. 12, pp. 705-710, 2005.

[96] R. Sanchez-De la Rosa, E. Sabater, and M. A. Casado, "Budget impact analysis of the first-line treatment of relapsing remitting multiple sclerosis in Spain," Revista de Neurología, vol. 53, no. 3, pp. 129-138, 2011.

[97] N. Scolding, "The multiple sclerosis risk sharing scheme," British Medical Journal, vol. 340, p. c2882, 2010.

[98] G. Stock and R. Horowski, "A short history of beta-interferon therapy of multiple sclerosis," Medizinische Klinik, vol. 96, supplement 1, pp. 3-9, 2001.

[99] P. Tappenden, J. B. Chilcott, S. Eggington, J. Oakley, and C. McCabe, "Methods for expected value of information analysis in complex health economic models: developments on the health economics of interferon- $\beta$ and glatiramer acetate for multiple sclerosis," Health Technology Assessment, vol. 8, no. 27, pp. 1-78, 2004.

[100] P. Tappenden, C. McCabe, J. Chilcott et al., "Cost-effectiveness of disease-modifying therapies in the management of multiple sclerosis for the medicare population," Value in Health, vol. 12, no. 5, pp. 657-665, 2009.

[101] P. Tappenden, R. Saccardi, C. Confavreux et al., "Autologous haematopoietic stem cell transplantation for secondary progressive multiple sclerosis: an exploratory cost-effectiveness analysis," Bone Marrow Transplantation, vol. 45, no. 6, pp. 1014-1021, 2010.

[102] A. J. Thompson, "Inequality in the availability of expensive treatments," Journal of the Royal College of Physicians of London, vol. 33, no. 5, pp. 448-449, 1999.

[103] K. H. Tolley and D. K. Whynes, "Interferon- $\beta$ in multiple sclerosis. Can we control its costs?" PharmacoEconomics, vol. 11, no. 3, pp. 210-215, 1997.
[104] D. R. Touchette, T. L. Durgin, L. A. Wanke, and D. E. Goodkin, "A cost-utility analysis of mitoxantrone hydrochloride and interferon beta-1b in the treatment of patients with secondary progressive or progressive relapsing multiple sclerosis," Clinical Therapeutics, vol. 25, no. 2, pp. 611-634, 2003.

[105] T. Walley, "Neuropsychotherapeutics in the UK: what Has Been the Impact of NICE on Prescribing?" CNS Drugs, vol. 18, no. 1, pp. 1-12, 2004.

[106] F. Pan, J. W. Goh, G. Cutter, W. Su, D. Pleimes, and C. Wang, "Long-term cost-effectiveness model of interferon Beta-1b in the early treatment of multiple sclerosis in the United States," Clinical Therapeutics, vol. 34, no. 9, pp. 1966-1976, 2012.

[107] J. P. Caloyeras, B. Zhang, C. Wang et al., "Cost-effectiveness analysis of interferon beta- $1 \mathrm{~b}$ for the treatment of patients with a first clinical event suggestive of multiple sclerosis," Clinical Therapeutics, vol. 34, no. 5, pp. 1132-1144, 2012.

[108] G. Kobelt, B. Texier-Richard, and P. Lindgren, "The longterm cost of multiple sclerosis in France and potential changes with disease-modifying interventions," Multiple Sclerosis, vol. 15, no. 6, pp. 741-751, 2009.

[109] P. Perini, M. Calabrese, M. Tiberio, F. Ranzato, L. Battistin, and P. Gallo, "Mitoxantrone versus cyclophosphamide in secondary-progressive multiple sclerosis: a comparative study," Journal of Neurology, vol. 253, no. 8, pp. 1034-1040, 2006.

[110] D. S. Goodin, E. M. Frohman, G. P. Garmany et al., "Disease modifying therapies in multiple sclerosis: report of the therapeutics and technology assessment subcommittee of the American academy of neurology and the MS council for clinical practice guidelines," Neurology, vol. 58, no. 2, pp. 169-178, 2002.

[111] National Collaborating Centre for Chronic Conditions, "Multiple sclerosis: management of multiple sclerosis in primary and secondary care," Tech. Rep., National Institue for Clinical Excellence, London, UK, 2003.

[112] M. C. Weinstein, B. O’Brien, J. Hornberger et al., "Principles of good practice for decision analytic modeling in health-care evaluation: report of the ISPOR task force on good research practices-modeling studies," Value in Health, vol. 6, no. 1, pp. 9-17, 2003. 


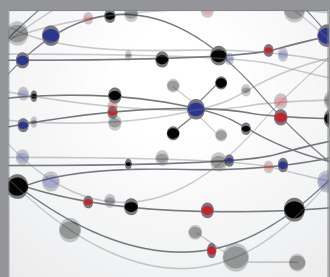

The Scientific World Journal
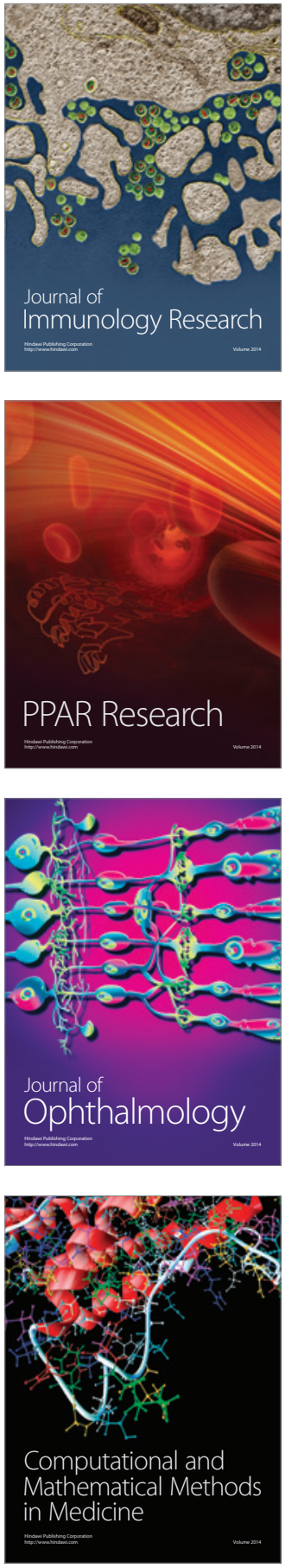

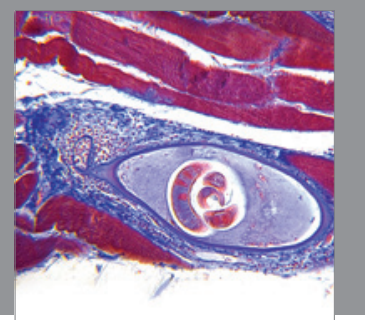

Gastroenterology

Research and Practice
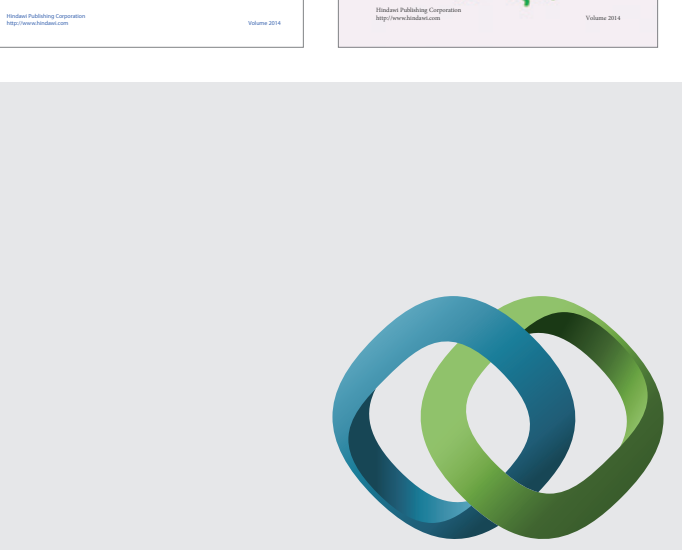

\section{Hindawi}

Submit your manuscripts at

http://www.hindawi.com
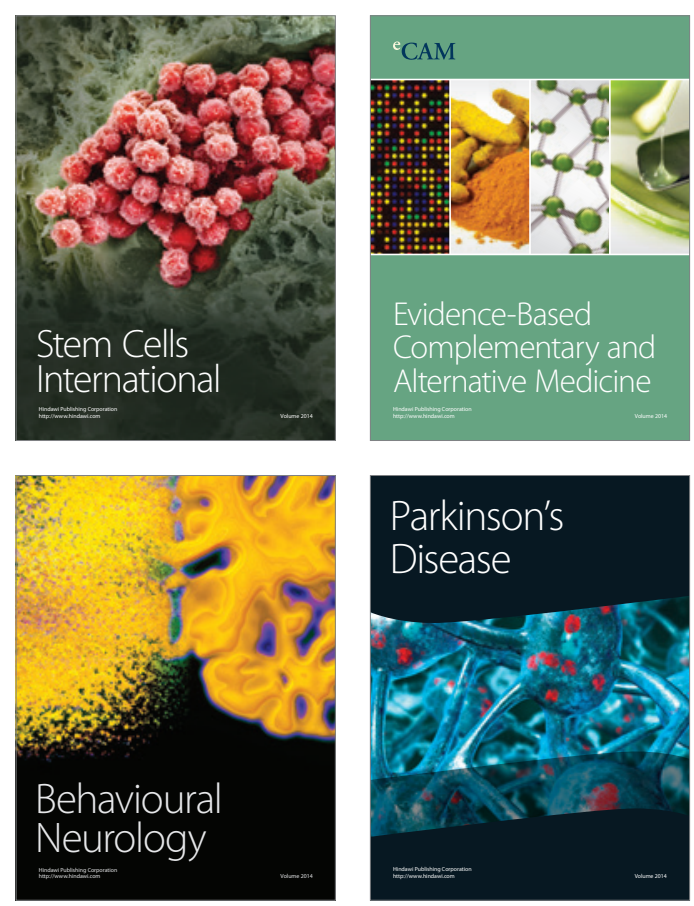

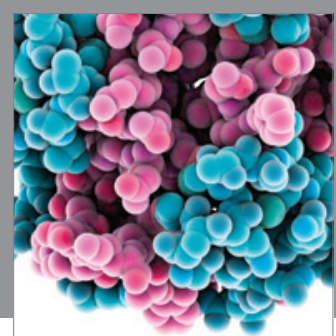

Journal of
Diabetes Research

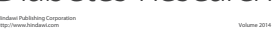

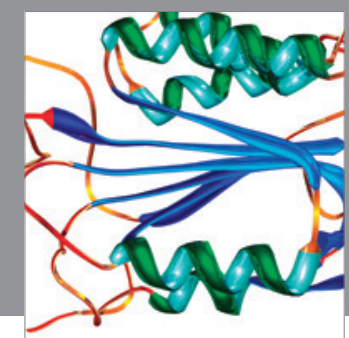

Disease Markers
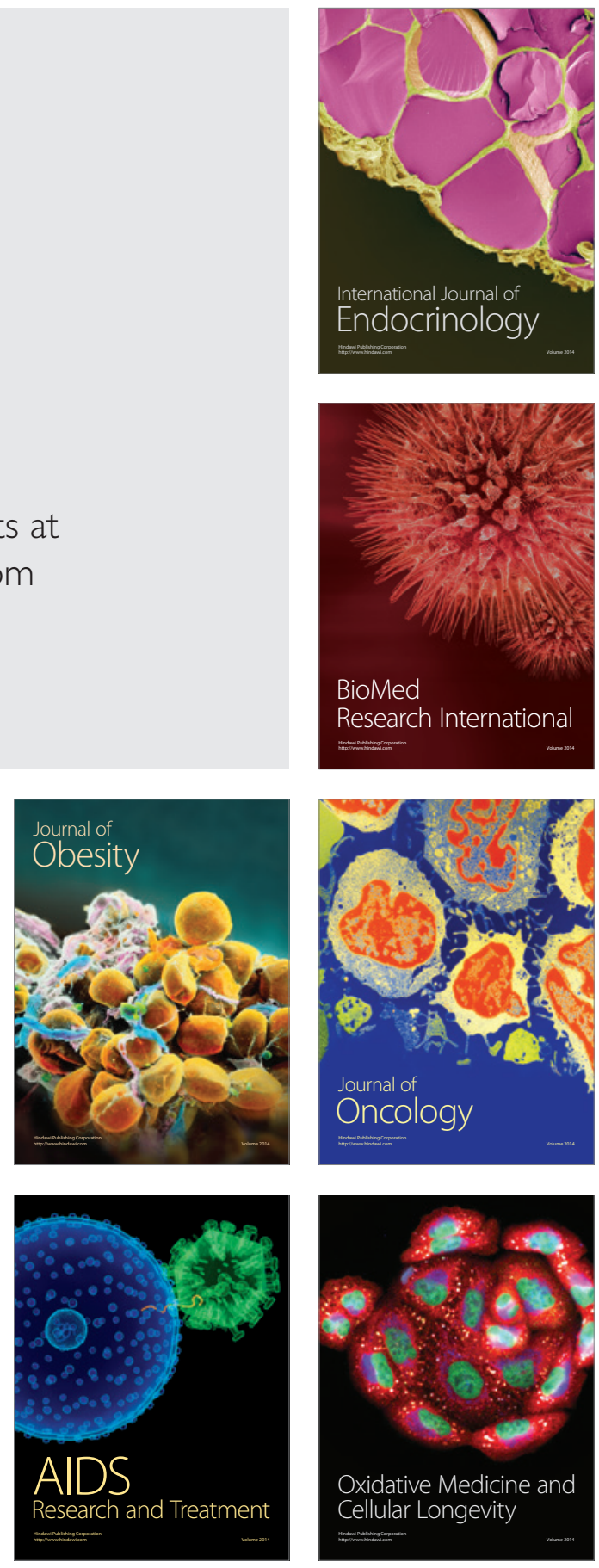